\title{
Lernen im Tandem in der bilingualen Erziehung
}

\section{Peter Doyé}

\section{OpenEdition}

\section{Journals}

Édition électronique

URL : http://journals.openedition.org/esp/425

DOI : $10.4000 /$ esp.425

ISSN : 2532-0319

\section{Éditeur}

Centre d'Information sur l'Éducation Bilingue et Plurilingue

\section{Édition imprimée}

Date de publication : 1 décembre 2014

Pagination : 17-24

ISSN : 1127-266X

\section{Référence électronique}

Peter Doyé, «Lernen im Tandem in der bilingualen Erziehung », Éducation et sociétés plurilingues

[Online], 37 | 2014, Online erschienen am: 01 Oktober 2015, besucht am 20 April 2019. URL : http:// journals.openedition.org/esp/425; DOI : 10.4000/esp.425 


\section{LERNEN IM TANDEM IN DER BILINGUALEN ERZIEHUNG}

\section{Peter Doyé}

(avec une présentation en français par Gilbert DALGALIAN)

Peter Doyé rimette la condivisione del sapere $e$ il mutuo insegnamento al centro della pedagogia delle lingue. Questo principio, valido in tutte le discipline, è particolarmente pertinente nel caso dell'apprendimento di una lingua viva. Qui le differenze fra alunni più competenti e alunni meno progrediti diventano un vantaggio per entrambe le parti. Gli esempi di tandem in ambito istituzionale sono sempre più numerosi, sia faccia a faccia che a distanza grazie alle nuove tecnologie. L'articolo espone dettagliatamente quattro illustrazioni pratiche in classe.

Peter Doyé replace le partage du savoir et l'enseignement mutuel au centre de la pédagogie des langues. Ce principe, valable dans toutes les disciplines, a une pertinence particulière dans l'apprentissage d'une langue vivante. C'est là que les différences entre élèves plus compétents et élèves moins avancés deviennent un atout pour les deux parties. Les exemples de tandems en milieu institutionnel sont de plus en plus nombreux, que ce soit en face-à-face ou à distance grâce aux nouvelles technologies. Quatre illustrations pratiques en classe sont détaillées.

Peter Doyé replaces knowledge sharing and reciprocal learning at the heart of language teaching. The principle is valid for all subject matters but is particularly pertinent for learning a spoken language, because the differences between the more competent and less advanced students then become an advantage for all sides. There are more and more examples of tandems in institutional contexts, either face to face or at a distance thanks to new technologies. Four illustrations of practical applications are given.

REFORMPÄDAGOGIK
I $m$ ersten Drittel des 20. Jahrhunderts gab es in Europa eine intensive pädagogische Diskussion um die als notwendig -erkannte Neuorientierung der Erziehung. Sie fand ihren Ausdruck in einer Reihe von Projekten von großer Nachhaltigkeit. Was man später als die „Reformbewegung" bezeichnete, hatte in den meisten europäsichen Ländern starke Auswirkungen auf die Entwicklung von Schule und Unterricht. Die „Schule des Lebens“ (Decroly), die „Produktionsschule“ (Blonsky), die „Schule der Selbsttätigkeit“ (Montessori), die 
Lernen im Tandem in der bilingualen Erziehung

P. DoYé
„Arbeitsschule“ (Gaudig und Kerschensteiner), die „École Moderne“ (Freinet), die „Reggio-Pädagogik“ (Malaguzzi) und die "Jenaplan-Schule" (Petersen), - sie alle beeinflussten in starkem Maße das spätere Denken und seine Praxis. So ist die Umgestaltung des deutschen Schulwesens nach dem zweiten Weltkrieg, besondern während der Mai-68-Bewegung, ohne den Rückgriff auf das Ideengut der zwanziger Jahre kaum denkbar. Man knüpfte ausdrücklich an die Vorschläge der Reformpädagogik an.

Die genannten Konzeptionen sind für die verschiedenen Bereiche des Lernens in der Schule entsprechend ihrer Ausrichtung von unterschiedlicher Bedeutung. So hat sich die „Arbeitsschule“ von Gaudig und Kerschensteiner als besonders fruchtbar für die Auseinandersetzung des Individuums mit seiner Umwelt erwiesen. Und die „École Moderne“ von Freinet hat gerade in Deutschland Vorbilder für die politische Bildung geliefert.

Hein Retter hat versucht, das Gemeinsame all dieser Konzeptionen zu eruieren und ist dabei zu der Erkenntnis gelangt, dass diese in der Ablehnung eines nur am Stoff orientierten Unterrichts zu sehen sei. „Stattdessen sollten Leben und Erleben, kindgerechte Aneignungsformen, letztlich das Kind und seine Selbsttätigkeit in den Mittelpunkt des Unterrichts gerückt werden." (Retter 1996, 162)

Ein besonders typisches Beispiel ist der Jenaplan von Peter Petersen. In ihm spielt das Konzept der Selbsttätigkeit eine zentrale Rolle. Was sein Verständnis dieser Idee aber für das Fremdsprachenlernen besonders interessant macht, ist ihre Ausprägung im Prinzip des gegenseitigen Helfens. „Die Möglichkeit des gegenseitigen Helfens und Sorgens“ sollte genutzt und zum Prinzip des Unterrichts gemacht werden und ein „Gefühl gegenseitiger Verantwortlichkeit“ geweckt werden. (Petersen 1966, 38/49)

Für die Fremdsprachendidaktik sind diese Vorschläge aus dem Jenaplan höchst relevant, allerdings nicht in fachspezifischer Weise, sondern als allgemeinpädagogische Grundlage. Was Petersen und seine Mitarbeiter dort konzipiert haben, ist eher als methodisches Prinzip von Bedeutung. In jeder Klasse, in jeder Gruppe gibt es starke und schwache Lernende; in jedem Sprachunterricht gibt es Aufgaben, die nur individuell bewältigt werden können. Nimmt man diese beiden Gedanken zusammen, dann wird klar, welchen Wert das Lernen in Kleingruppen, am besten in sogenannter Partnerarbeit haben kann. „Individuell“ ist 
Lernen im Tandem in der bilingualen Erziehung

P. Doyé

SPRACHENLERNEN IM TANDEM bei Petersen nicht gleichbedeutend mit „einzeln“, sondern schließt das Lernen in Kleingruppen ein, vor allem das Lernen in Zweiergruppen.

Das Lernen in Zweiergruppen mit dem ausdrücklichen Ziel der gegenseitigen Unterstützung der Partner ist in neuerer Zeit von einer Gruppe Pädagogen aufgegriffen und zu einem didaktischen Prinzip erhoben worden. Der Grundgedanke ist der, dass die Lernenden ihre eigene Kompetenz zur Förderung des Lernens ihres jeweiligen Partners einsetzen. Jeder der beiden ist auf einem bestimmten Gebiet dem anderen überlegen und setzt diese Überlegenheit zur Hilfe für den anderen ein. Dadurch, dass das Unternehmen auf Gegenseitigkeit beruht, entwickelt sich eine echte Lernpartnerschaft, im Idealfall ein Gefühl der gegenseitigen Verantwortung und Solidarität.

Diese pädagogischen Annahmen sind psychologisch gut begründet. Unter Sozialpsychologen herrscht Einigkeit darüber, dass die Zweierbeziehung die intensivste aller Beziehungen darstellt und dass die dyadische Interaktion Vorteile besitzt, die keine andere Interaktion bieten kann.

,Jeder Partner kommt zu der Begegnung auf Grund von Motivationen, die durch das Geschehen in der Begegnung befriedigt werden können; Motivation lässt sich hier in Termini der Ziele betrachten, die ein Individuum zu erreichen trachtet; sie bestehen entweder in bestimmten Verhaltensweisen des anderen (z.B. Bewunderung) oder in einem Muster dyadischer Interaktion (z.B. Intimität).“ (Argyle 1995: 163)

Anwendung gefunden haben diese Gedanken in dem Konzept des Sprachenlernens im Tandem. Da alles Fremdsprachenlernen ohnehin der Bewältigung von Kommunikationssituationen mit Menschen anderer sprachlicher und kultureller Herkunft dient, lag es nahe, solche Situationen schon im Lernprozess selbst zu schaffen, wo immer dies praktisch möglich ist. Menschen verschiedener Erstsprachen, die die Sprachen von Personen anderer Provenienz erlernen wollen oder sollen, werden zusammengeführt und angeleitet, sich mit Hilfe ihrer Partner deren Sprache anzueignen und ihnen bei der Aneignung der eigenen Sprache zu helfen.

„Beim Sprachenlernen im Tandem kommunizieren zwei Personen mit unterschiedlichen Muttersprachen, um gemeinsam und voneinander zu lernen.

Beide suchen dabei,

- ihre Kommunikationsfähigkeit in der Muttersprache des 


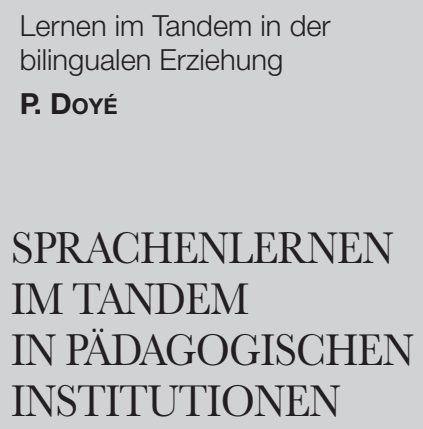

Partners zu verbessern,

- mehr über die Person und den kulturellen Hintergrund des Partners zu erfahren sowie

- von anderen Kenntnissen und Erfahrungen des Partners zu profitieren. (Brammerts \& Kleppin 2010: 10).

Nun sind Situationen, in denen Partner für das Tandem-Lernen zur Verfügung stehen, in europäischen Bildungssystemen nicht gerade die Regel. Hieraus erklärt sich die geringe Verbreitung des so plausiblen Prinzips.

Praktiziert wird das Tandem-Lernen bisher hauptsächlich in der individuellen Vermittlung von Partnerschaften, in Hochschulen, in mehrsprachigen Schulen und in bilingualen vorschulischen Einrichtungen.

Die individuelle Vermittlung von Partnerschaften über Sprachgrenzen hinweg war die ursprüngliche Form des Tandem-Lernens in der Sprachaneignung. Private oder auch staatliche Institutionen nahmen sich der Betreuung von Personen an, die eine andere Sprache lernen wollten, und führten Menschen jeweils passender Herkunft zusammen. Die Institutionen beschränkten sich zunächst auf die Herstellung von Kontakten und organisatorische Aufgaben. Mittlerweile unterstützen die Vermittler die Lernenden aber auch methodisch und beraten sie bei ihren Bemühungen.

$\mathrm{Zu}$ unterscheiden sind das sogenannte Präsenztandem und das Distanztandem.

In ersterem treffen sich Lernende am gleichen Ort und lernen face to face. Die meisten Face-to-face-Paare arbeiten kursunabhängig und nutzen ihre Partnerschaften selbstständig und individuell. In letzterem kommunizieren die Partner per Post oder über elektronische Medien. Die starke Verbreitung der neuen Medien erleichtert diese Form enorm und ist dabei, die traditionelle Form der Partnerschaft per Post zu verdrängen.

In den vergangenen Jahren hat das Tandem-Lernen nun auch Eingang in verschiedene Studiengänge an Hochschulen gefunden. Brammerts und Kleppin haben in ihrem Standardwerk aufschlussreiche Berichte von Hochschulkursen zusammengestellt, in denen das Tandem-Lernen in die fremdsprachlichen Curricula integriert wurde. (Brammerts \& Kleppin 2010, 123ff.) Solche Kurse gibt es in Sheffield, Bochum, Oviedo, Coimbra und Guadalajara.

Am stärksten vertreten ist das Tandem-Lernen aber an internationalen Schulen, also Schulen, die sich schon von der Zusammensetzung ihrer Schülerschaft her für diese Form des 
Lernen im Tandem in der bilingualen Erziehung

P. DoYÉ
Sprachenlernens anbieten. Ein Musterbeispiel ist die Staatliche Europaschule Berlin (SESB). In ihr gibt es an 18 Standorten bilinguale Einrichtungen mit insgesamt neun Sprachkombinationen, an denen Schülerinnen und Schüler mit deutscher Muttersprache gemeinsam mit Kindern und Jugendlichen je einer anderen Muttersprache erzogen werden. Es gibt deutsch-polnische, deutsch-russische, deutsch-englische, deutsch -französische, deutsch-italienische, deutsch-spanische, deutsch-portugiesische, deutsch-türkische und deutsch-griechische Standorte. In all diesen Einrichtungen arbeiten deutsche Lehrkräfte gemeinsam mit solchen der jeweils anderen Partnersprache zusammen. Wichtiger ist aber die Bikulturalität der Schülerschaft. Die Organisatoren achten darauf, dass in jeder Klasse ein ausgewogenes Verhältnis zwischen den deutschen Schülern und denen der assoziierten anderen Kultur und Sprache besteht, im Idealfall in gleicher Zahl. So ergibt sich eine optimale Voraussetzung für das Lernen im Tandem.

Eine ähnliche Situation besteht in Wolfsburg, wo in der Leonardo-da-Vinci-Schule deutsche und italienische Kinder und Jugendliche gemeinsam erzogen werden und die Lehrkräfte ebenfalls die Chancen des Tandem-Lernens nutzen, wo immer das möglich ist.

Das Fazit: Überall, wo in der SESB und ähnlich gelagerten Schulen nach dem Tandem-Prinzip gelernt und gelehrt wurde, sind die Ergebnisse beachtlich und in in mehreren Studien gut dokumentiert worden. (Göhlich 1997)

Weniger Beachtung haben in der pädagogischen Öffentlichkeit die bilingualen Kindergärten gefunden, in denen das TandemLernen ebenso gute Erfolge gebracht hat. Solche Einrichtungen gibt es z. B. in Berlin, Kiel, Köln, München und Wolfsburg. (Doyé 2009) Sie sind geschaffen worden, um Kindern, die aus anderen Ländern nach Deutschland eingewandert sind, Hilfen bei ihrer Integration zu geben. Viele dieser Kinder sprechen unzureichend Deutsch und sind daher für hiesige Verhältnisse nicht schulreif. Für sie ist die gemeinsame Erziehung mit deutschen Kindern eine optimale Möglichkeit zur Integration. Fthenakis (1985) nennt die nach diesem Modell arbeitenden Curricula „Emanzipationsprogramme“ oder „Integrationsprogramme“. Das Besondere an ihnen ist - im Gegensatz zu sonst üblichen „Bereicherungsprogrammen“ -- die Gleichgewichtung zweier Prinzipien: Die Integration wird gefördert, ohne dass die Kinder ihre Erstsprache aufgeben müssen. (Fthenakis 1985). Die beiden Kulturen - die des Aufnahmelandes wie die des Herkunftlandes - 
Lernen im Tandem in der bilingualen Erziehung

P. DOYÉ

BEISPIELE FÜR DAS TANDEM-LERNEN AN BILINGUALEN SCHULEN

\section{BEISPIEL 1:}

BEISPIEL 2: werden gleichermaßen gepflegt, was übrigens diese Kindergärten auch für deutsche Eltern attraktiv macht. Mit anderen Worten: Beide Seiten profitieren von der Zusammenführung. Die gemeinsame Erziehung schafft eine optimale Grundlage für das Tandem-Lernen.

Französischunterricht in einer 2. Klasse der SESB.

Intention: Verständnis einer französischen Kurzgeschichte

Die Lehrerin erzählt den französischsprachigen Kindern eine humorvolle Geschichte, die ihnen sehr gefällt. Sie äußert das Bedauern darüber, dass die deutschen Klassenkameraden die Geschichte nicht hören konnten und mangels Sprachkenntnis wohl auch nicht verstanden hätten.

In einem gelenkten Gespräch werden Maßnahmen besprochen, die den deutschsprachigen Kindern das Verständnis ermöglichen könnten. Der Vorschlag, Bilder zu den Schlüsselszenen zu malen, wird angenommen. Jedes französischsprachige Kind zeichnet nun solche Szenen für seinen deutschen Partner, der daraufhin die Geschichte bei nochmaligem Erzählen versteht.

In der nächst folgenden Stunde organisiert die Deutschlehrerin ein entsprechendes Vorgehen, bei dem die deutschen Kinder ihren französischen Partnern anhand von Zeichnungen beim Verstehen einer deutschen Geschichte helfen.

Sachunterricht in einer 4. Klasse der Leonardo-da-Vinci-Schule, Wolfsburg

Thema: Kleidung

Die Lehrerin hat den Kindern Versandkataloge zur Verfügung gestellt, aus denen sie sich Kleidungsstücke heraussuchen, die ihnen gefallen, - italienische Kataloge für die italienischsprachigen Kinder und deutsche Kataloge für die deutschsprachigen.

Alle Kinder stellen sich ein „Kostüm“ aus ausgewählten Stücken zusammen und beschreiben es in ihrer Sprache für ihre TandemPartner. Sodann wechseln sie ihre Rollen und beschreiben die Kostüme ihrer Partner in deren Sprache. Auf diese Weise erwerben sie das Vokabular des Bereichs „Kleidung" in der Partnersprache, wobei gegenseitige Hilfe angesagt ist.

Schließlich bereiten beide Kinder jeder Zweiergruppe gemeinsam ihren Auftritt in einer Sfilata di moda vor, an der alle Kinder der Klasse teilnehmen. 


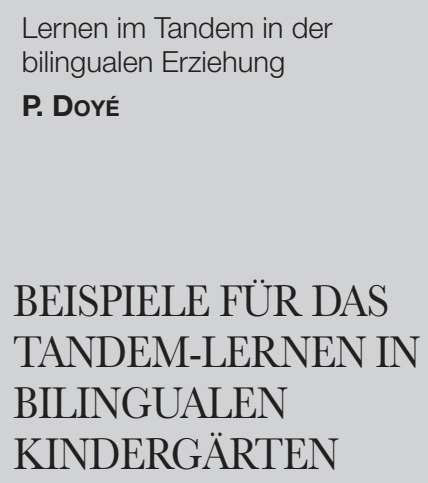

BEISPIEL 3:

BEISPIEL 4:
Umweltkunde in einer deutsch-russischen Kindertagesstätte in Braunschweig

Thema: Briefmarken

Jedes Kind hat 5 bis 8 Briefmarken seines Landes mitgebracht und sich zu jeder von ihnen einige kurze Äußerungen ausgedacht. Es zeigt seinem Tandem-Partner die einzelnen Briefmarken und liefert die vorbereiteten Kommentare dazu. Diese enthalten lernenswertes Vokabular, die der Partner in seinen Wortschatz aufnimmt. Die beiden kooperierenden Kinder helfen einander, indem sie sich gegenseitig die Bedeutung der Wörter erklären und deren Aussprache und Schreibweise demonstrieren.

Anschließend werden Bedeutung, Aussprache und Schreibweise individuell geübt, wofür gerade die Konstitution der Zweiergruppe eine günstige Voraussetzung bietet.

Spielerisches Lernen in einer deutsch-englischen Kindertagesstätte in Wolfsburg

Thema: Rätselraten

Die Erzieherin wiederholt in spielerischer Form die Bezeichnungen wichtiger Gegenstände der unmittelbaren Umwelt in beiden Sprachen. Sodann stellt sie den Kindern das Rätsel „I spy with my little eye“ bzw. „Ich sehe was, was du nicht siehst" vor und erklärt ihnen die Regeln. Danach geben die Kinder jeder Zweiergruppe ihren Partnern Rätsel in ihrer Sprache auf, die diese zu lösen versuchen. Dies geschieht nacheinander und abwechselnd, wobei das jeweils verwendete Vokabular entweder gefestigt oder neu vermittelt wird.

\section{ZUSAMMENFASSUNG}

Was ist das Gemeinsame in diesen vier Beispielen?

Stets sind Tandem-Lerngruppen am Werk, die eine gemeinsame oder parallele Aufgabe lösen. Durch Impulse und Hilfestellungen unterstützen die beiden Partner einer jeden bilingualen Zweiergruppe einander beim Sprachenlernen durch Impulse und Hilfestellungen.

.Das Tandem-Prinzip bietet drei Vorteile:

Die Kinder beider Sprachgruppen profitieren von der höheren sprachlichen Kompetenz der jeweils anderen Gruppe, wenn sie deren Muttersprache als Zweitsprache lernen.

Sie werden sich ihrer eigenen muttersprachlichen Kompetenz bewusst, wenn sie Kinder der anderen Gruppe bei deren Bemühen um eine zweite Sprache unterstützen, die ihre Muttersprache ist. 
Lernen im Tandem in der bilingualen Erziehung

P. Doyé
Sie erkennen, dass die jeweilige Ungleichheit in den sprachlichen Voraussetzungen durch gegenseitige Hilfe ausgeglichen werden kann.

Alle drei Vorteile sind von großer didaktischer Relevanz. Der letztgenannte Punkt ist darüber hinaus von allgemein-pädagogischer Bedeutung: Die Einsicht, dass ungleiche Kompetenzen in verschiedenen Leistungsbereichen durch gegenseitige Unterstützung überwunden werden können, ist eine wichtige Voraussetzung für die Bereitschaft junger Menschen zur Kooperation.

ARGYLE M. 1975, 3. Aufl. Soziale Interaktion. Köln: Kiepenheuer \& Witsch.

BRAMMERTS H. \& KLEPPIN K. 2010, 3. Aufl. Selbstgesteuertes Lernen im Tandem. Tübingen: Stauffenberg.

DALGALIAN G. 2000. Enfances plurilingues. Témoignage pour une éducation bilingue et plurilingue. Paris: L'Harmattan.

DALGALIAN G. 2012. Reconstruire l'éducation ou Le désir d'apprendre. Paris: Insititut de recherches de la FSU/Syllepse.

DOYÉ P. 2009. Didaktik der bilingualen Vorschulerziehung. Tübingen: Gunter Narr Verlag.

DOYÉ P., MANAZZA A. \& POSILLICO F. 2011. Vivere due lingue. Italienisch im bilingualen Kindergarten. Hildesheim: Georg Olms Verlag.

DOYE P. \& JERECZEK C. 2012. Enfance bilingue. Französisch im bilingualen Kindergarten. Hildesheim: Georg Olms Verlag.

FTHENAKIS W. et al. 1985. Bilingual-bikulturelle Entwicklung des Kindes. München: Max Hueber Verlag.

GÖHLICH M. (Hrsg.). 1998. Europaschule. Das Berliner Modell. Neuwied: Luchterhand.

PETERSEN P. 1970, 9. Aufl. Führungslehre des Unterrichts. Braunschweig.

PETERSEN P. 1968, 51. Aufl. Der kleine Jenaplan. Weinheim \& Berlin: Julius Beltz.

RETTER H. 1996. Reformpädagogik zwischen Rekonstruktion, Kritik und Verständigung. Weinheim: Deutscher Studienverlag.

RICGÓ A. \& SANDFUCHS U. (Hrsg.). 1996. Insieme Gemeinsam. Bad Heilbrunn: Klinkhardt.

SCHMELTER L. 2010. Tandemlernen. In: HALLET W. \& KÖNIGS F. G. (Hrsg.). Handbuch Fremdsprachendidaktik. SeelzeVelber: Klett Kallmeyer. 